\title{
Negative magnetoresistance in epitaxial films of neodymium nickelate
}

\author{
A. Stupakov, ${ }^{1}$ O. Pacherova, ${ }^{1}$ T. Kocourek, ${ }^{1}$ M. Jelinek, ${ }^{1}$ A. Dejneka, ${ }^{1}$ and M. Tyunina ${ }^{1,2, *}$ \\ ${ }^{1}$ Institute of Physics of the Czech Academy of Sciences, Na Slovance 2, 18221 Prague, Czech Republic \\ ${ }^{2}$ Microelectronics Research Unit, Faculty of Information Technology and Electrical Engineering, University of Oulu, P.O. Box 4500, \\ FI-90014 Oulu, Finland
}

(Received 22 November 2018; revised manuscript received 21 January 2019; published 7 February 2019)

\begin{abstract}
A significant increase in conductivity under an applied magnetic field or large negative magnetoresistance up to several percent is observed at low temperatures in epitaxial perovskite $\mathrm{NdNiO}_{3}$ films. The $(20-100 \mathrm{~nm})$ strained films are grown on compressive $(001) \mathrm{LaAlO}_{3}$ and tensile $(001)\left(\mathrm{La}_{0.3} \mathrm{Sr}_{0.7}\right)\left(\mathrm{Al}_{0.65} \mathrm{Ta}_{0.35}\right) \mathrm{O}_{3}$ substrates. The negative magnetoresistance is found in the insulating phase, where the hopping mechanism of conductivity is revealed. It is shown that the presence and Zeeman splitting of localized states are responsible for conductivity and magnetoresistance. The localized states are suggested to emerge due to thermally induced local disorder in the Ni-O bond disproportionation. Such disorder can also lead to a phase coexistence in a broad temperature range.
\end{abstract}

DOI: 10.1103/PhysRevB.99.085111

\section{INTRODUCTION}

Perovskite-structure neodymium nickelate $\mathrm{NdNiO}_{3}(\mathrm{NNO})$ is the best studied representative of the rare-earth nickelates, exhibiting a sharp metal-to-insulator transition (MIT) on cooling. The high sensitivity of the electrical, magnetic, and optical properties of nickelates to external stimuli such as temperature, electric and/or magnetic fields, light, and ambient chemistry can enable innovative electronics applications that motivates the extensive research of the complex physics and synthesis of these materials. In particular, single-crystaltype epitaxial nickelate films attract worldwide research efforts because such films are the most promising for modern miniature applications, ensure perovskite-pure materials for fundamental investigations, and allow for controlling the film properties using epitaxial strain and interfacial phenomena. The progress in the synthesis and understanding of nickelates is summarized in a number of reviews, including the most cited and most recent ones [1,2].

The key issue of fundamental and practical importance is the MIT. According to modern knowledge, the MIT is associated with a structural transition from the high-temperature orthorhombic Pbmn phase, exhibiting metal conductivity, to the low-temperature monoclinic $P 2_{1} / n$ phase, possessing insulating properties. The insulating phase is characterized by a $\mathrm{Ni}-\mathrm{O}$ bond length disproportionation, where neighboring $\mathrm{Ni}$ sites possess different bond lengths and are inequivalent: $\mathrm{Ni}_{S}$ (the shorter bond) and $\mathrm{Ni}_{\mathrm{L}}$ (the longer bond). The small $\mathrm{Ni}_{\mathrm{S}} \mathrm{O}_{6}$ and large $\mathrm{Ni}_{\mathrm{L}} \mathrm{O}_{6}$ octahedra are ordered in the rock-salt pattern. As shown theoretically, the distinct $\mathrm{Ni}-\mathrm{O}$ bonds are pivotal for the MIT [3-6]. It was also shown that the insulating phase may be sensitive to magnetic ordering [6]. Unfortunately, the temperature-dependent behavior has not been captured by theoretical calculations so far.

\footnotetext{
*Corresponding author: marina.tjunina@oulu.fi
}

Here, we focus on the temperature- and magnetic-fielddependent properties of the insulating phase in epitaxial NNO films. By experimental studies of conductivity as a function of temperature and applied magnetic field, we detect a significant low-temperature magnetoresistance. The results are analyzed using models of hopping conductivity and evidence the presence and Zeeman splitting of localized states. The analysis shows that the gap filling with states, previously found on approaching the MIT, starts at very low temperatures. A thermally induced local disorder in bond disproportionation is discussed as responsible for the localized states and for phase coexistence in a broad temperature range. We anticipate that our findings will boost better understanding and control of the MIT.

\section{EXPERIMENT}

Thin films of $\mathrm{NdNiO}_{3}$ (NNO) of $20-100 \mathrm{~nm}$ in thickness were deposited using single-crystal epitaxially polished (001) $\mathrm{LaAlO}_{3}$ (LAO) and (001) $\left(\mathrm{La}_{0.3} \mathrm{Sr}_{0.7}\right)\left(\mathrm{Al}_{0.65} \mathrm{Ta}_{0.35}\right) \mathrm{O}_{3}$ (LSAT) substrates purchased from the MTI Corporation. The films were grown by pulsed laser deposition (PLD) using a $\mathrm{KrF}$ excimer laser (energy density $\sim 2 \mathrm{~J} / \mathrm{cm}^{2}$ ) at a substrate temperature of $973 \mathrm{~K}$ and ambient oxygen pressure of $20 \mathrm{~Pa}$. The employed high oxygen pressure ensures the growth of stoichiometric defect-free perovskite films, in contrast to the defect formation that occurs at lower pressures $[7,8]$. To further secure proper oxygen stoichiometry, the oxygen pressure was gradually increased to $800-1000 \mathrm{~Pa}$ during the post-deposition cooling, which was conducted at a rate of $5 \mathrm{~K} / \mathrm{min}$.

The crystal structure of the films was studied by highresolution $\mathrm{x}$-ray diffraction (XRD) on a D8 Discover diffractometer (Bruker Corporation) using $\mathrm{Cu} \mathrm{K} \alpha$ radiation. $\Theta-2 \Theta$ scans in the range of $2 \Theta=(10-130)$ deg and reciprocal space maps (RSM) in the vicinity of the perovskite (002), (303), and (103) diffractions were acquired. The in-plane 
(parallel to the substrate surface) and out-of-plane (normal to the substrate surface) lattice parameters were estimated from the positions of the diffraction maxima using the substrates as a reference.

For electrical characterization, four equally separated $\mathrm{Au}$ top contact stripes of $1 \mathrm{~mm}$ in width were formed by the room-temperature vacuum PLD of $\mathrm{Au}$ through a shadow mask. The contacts were oriented along the [100] (or [010]) substrates crystal direction, parallel to the shorter cut of the samples, which were $4 \times 10 \mathrm{~mm}$ in size. The Au wires were attached to the $\mathrm{Au}$ top contacts using colloidal $\mathrm{Au}$ paste.

Four-probe measurements of the electrical resistivity were performed on a Physical Property Measurement System (PPMS) from Quantum Design using dc/ac current-controlled modes. The measurements were carried out at varying magnitudes (up to $10 \mathrm{~T}$ ) and directions of the dc magnetic field. The resistivity was studied on cooling, heating, and at several fixed temperatures in a range of $2-400 \mathrm{~K}$. For a fixed temperature, the magnetic field was swept at a rate of $100 \mathrm{Oe} / \mathrm{s}$. A heating/cooling rate of $2 \mathrm{~K} / \mathrm{min}$ was used in the measurements both with and without the magnetic field.

\section{RESULTS AND DISCUSSION}

\section{A. Film structure}

A room-temperature crystal structure of bulk unstressed NNO is orthorhombic (Pbnm) with lattice parameters of $0.5389,0.5382$, and $0.7610 \mathrm{~nm}$ [9]. The orthorhombic unit cell is formed by tilted perovskite subcells with lattice parameters of $0.3808,0.3805$, and $0.3808 \mathrm{~nm}$. Considering a cube-on-cube pseudomorphic epitaxial growth of perovskite NNO on substrates with a square surface lattice, an average lattice parameter $a_{\mathrm{NNO}}=0.3807 \mathrm{~nm}$ is usually employed to estimate the NNO-substrate lattice-parameter misfit strain $s_{a}$ as follows: $s_{a}=\left(a_{\mathrm{SUB}} / a_{\mathrm{NNO}}-1\right)$, where $a_{\mathrm{SUB}}$ is the lattice parameter on the substrate surface. The latticeparameter misfit is compressive $\sim 0.4 \%$ in $\mathrm{NNO} / \mathrm{LAO}$ and tensile $\sim 1.6 \%$ in NNO/LSAT. Additionally, there is a symmetry misfit arising from $\mathrm{NiO}_{6}$-octahedral tilts (rotations) in NNO. Such a symmetry misfit is typical for the epitaxy of orthorhombic perovskites on square surfaces of versatile substrates [10-13]. The symmetry misfit relaxes within a few nanometers near the film-substrate interface, as was evidenced by the evolution of tilts towards bulklike patterns in nickelates [11,14-16].

Our XRD observations can be interpreted in terms of highly oriented perovskite NNO films, with the $(00 l)$ planes of the perovskite cell $\left(\mathrm{NNO}_{\mathrm{p}}\right)$ parallel to the substrate surface (Fig. 1). The Laue satellite diffractions confirm high crystal quality and surface smoothness of the films [Figs. 1(b) and 1(g)]. Also, cube-on-cube-type epitaxial relationships $[100](001) \mathrm{NNO}_{\mathrm{p}} \|[100](001)$ substrate can be concluded [Figs. 2(a) and 2(b)]. The in-plane lattice parameters of the films are approximately equal to those of the underlying substrates. The out-of-plane lattice parameters are $\sim(0.3794-$ $0.3805) \mathrm{nm}$ and $\sim(0.3840-0.3845) \mathrm{nm}$ in the (20-100)-nmthick films on LAO and LSAT, respectively. The epitaxial relationships and measured lattice parameters agree with the previous observations [11,15-21]. We note that a few-nanometerthick interfacial layer, whose crystal and electronic structures differ from those inside the body of the films, cannot be detected by laboratory XRD. As shown before, NNO films accommodate epitaxial strain through additional (compared to bulk) Ni-O bond elongations and contractions while adopting the bulklike tilt pattern. This ability is manifested in nearly similar strain states detected in the 20- and 100-nm-thick films: otherwise, efficient strain relaxation (e.g., through the formation of dislocations) would have been observed with increasing film thickness.

Unlike the bulk NNO perovskite cell, the NNO/LAO films possess cells, which are compressed in the two in-plane directions and elongated in the out-of-plane direction [Fig. 1(e)] and the NNO/LSAT films possess cells, which are elongated in the two in-plane directions and compressed in the out-ofplane direction [Fig. 1(j)].
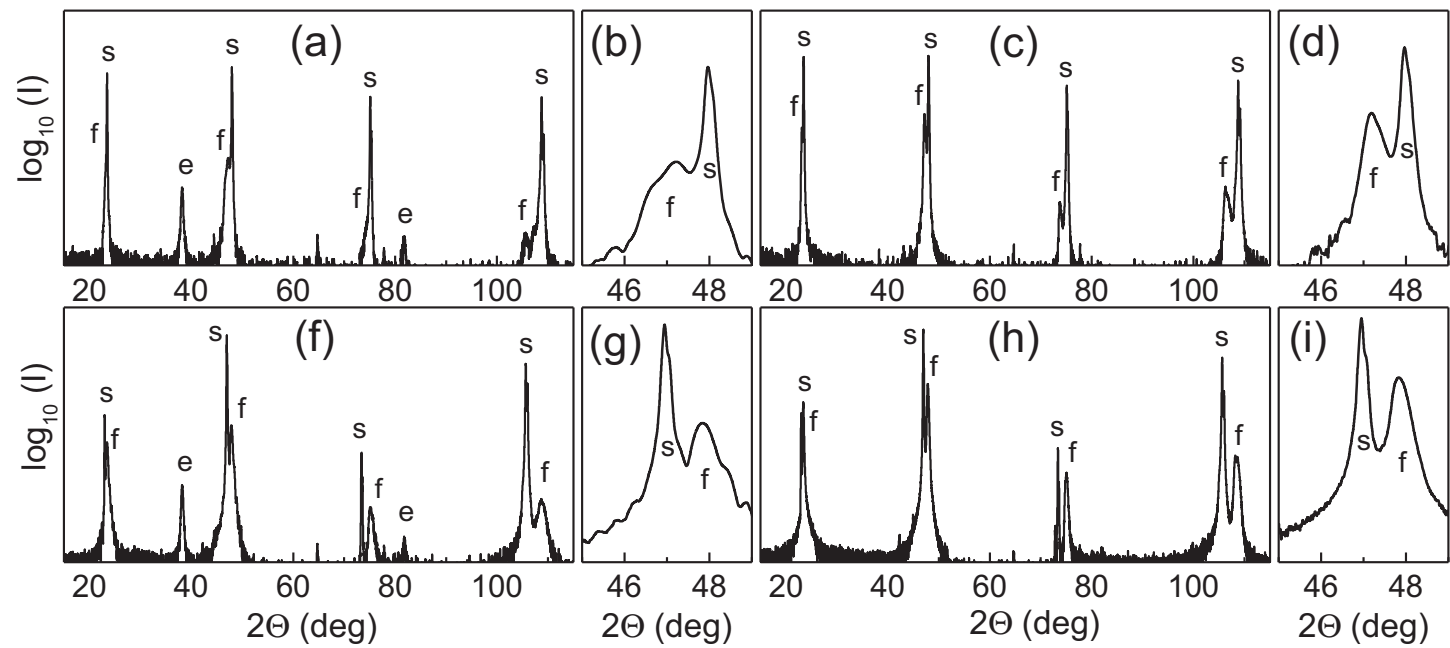

4060
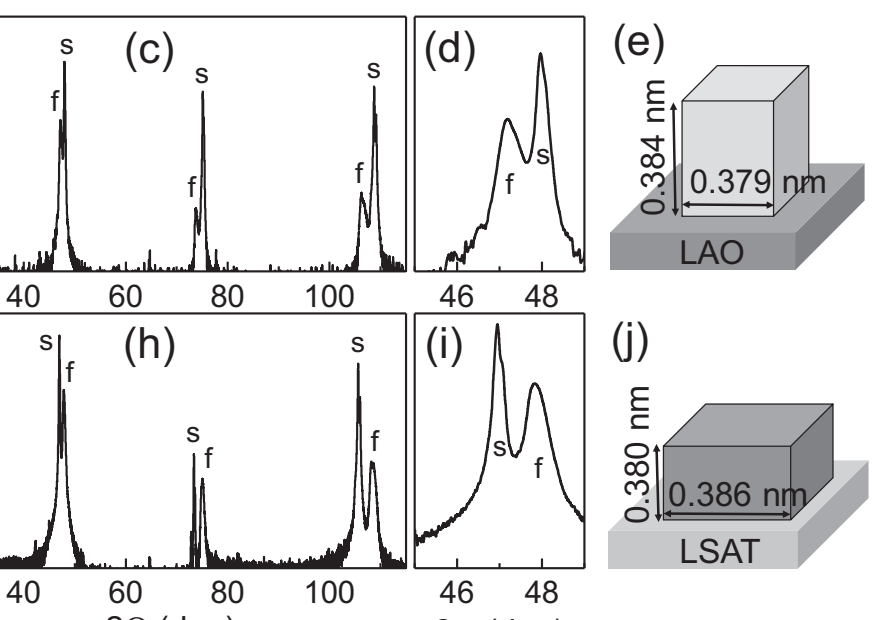

FIG. 1. XRD $\Theta-2 \Theta$ patterns in the NNO films on (a)-(d) LAO and (f)-(i) LSAT. Thicknesses are (a),(b),(f),(g) $20 \mathrm{~nm}$ and (c),(d),(h),(i) $100 \mathrm{~nm}$. (b),(d),(g),(i) The details around the (002) perovskite diffraction. Symbols "s", "f," and "e" mark diffractions from the substrates, films, and Au electrodes, correspondingly. (e),(j) Schematics of perovskite NNO cells in the films on (e) LAO and (j) LSAT. 

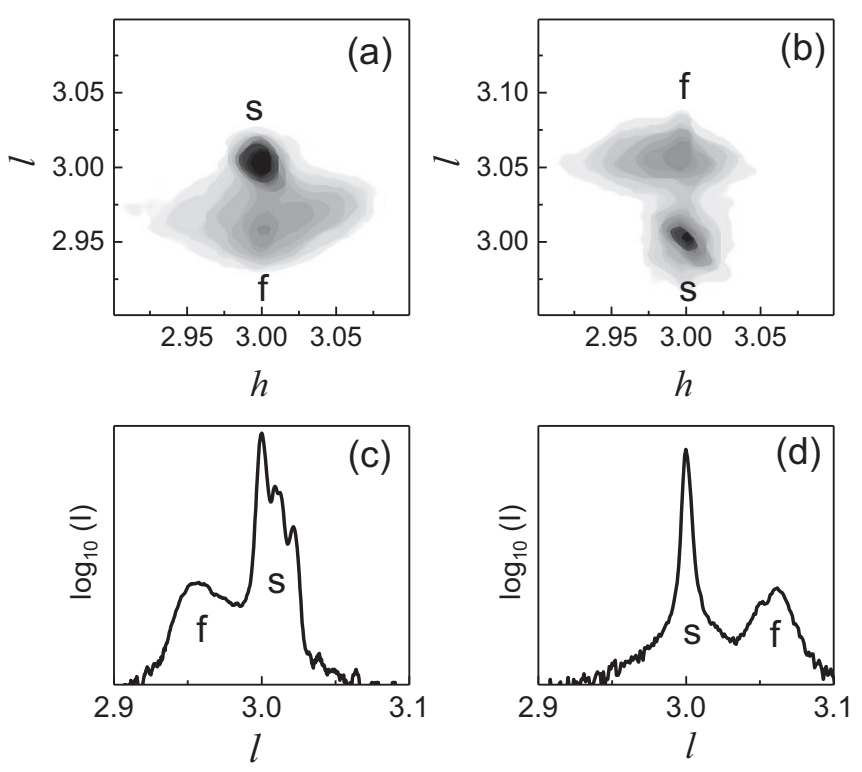

FIG. 2. (a),(b) Reciprocal space maps around the (303) reciprocal lattice points and (c),(d) scans along the (303) reciprocal lattice points ( $h=3, k=0)$ in the 100-nm-thick NNO films on (a),(c) LAO and (b),(d) LSAT substrates. Data are presented in the reciprocal lattice units of the substrates. Symbols "s" and "f" mark diffractions from the substrates and NNO films, respectively.

\section{B. Resistivity}

The magnitude and temperature evolution of the resistivity $\rho$ in our NNO films (Fig. 3) are in good agreement with the previous works [18-21]. Compared to the bulk MIT at the temperature $T_{\mathrm{MI}}=200 \mathrm{~K}$, the increase in resistivity on cooling appears to be suppressed and the temperature $T_{\mathrm{MI}}$ appears to be significantly lowered in the NNO/LAO films [Figs. 3(a)
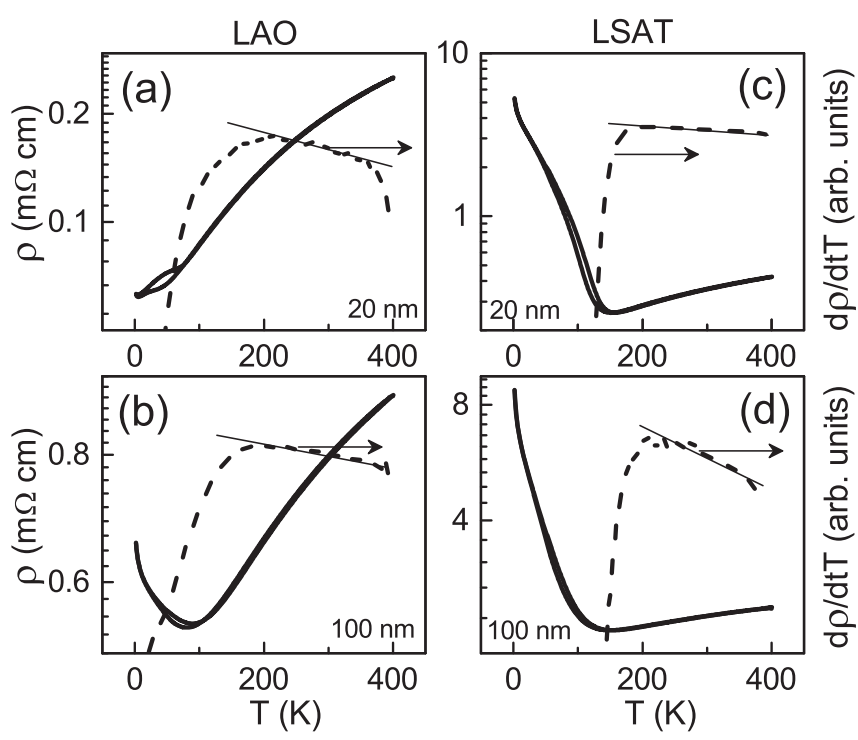

FIG. 3. Resistivity $\rho$ (on a logarithmic scale) as a function of temperature in the NNO films on (a),(b) LAO and (c),(d) LSAT. Film thicknesses are (a),(c) $20 \mathrm{~nm}$ and (b),(d) $100 \mathrm{~nm}$. Dashed curves show the derivative $d \rho / d T$. Thin straight lines in $d \rho / d T$ indicate the metal phase.

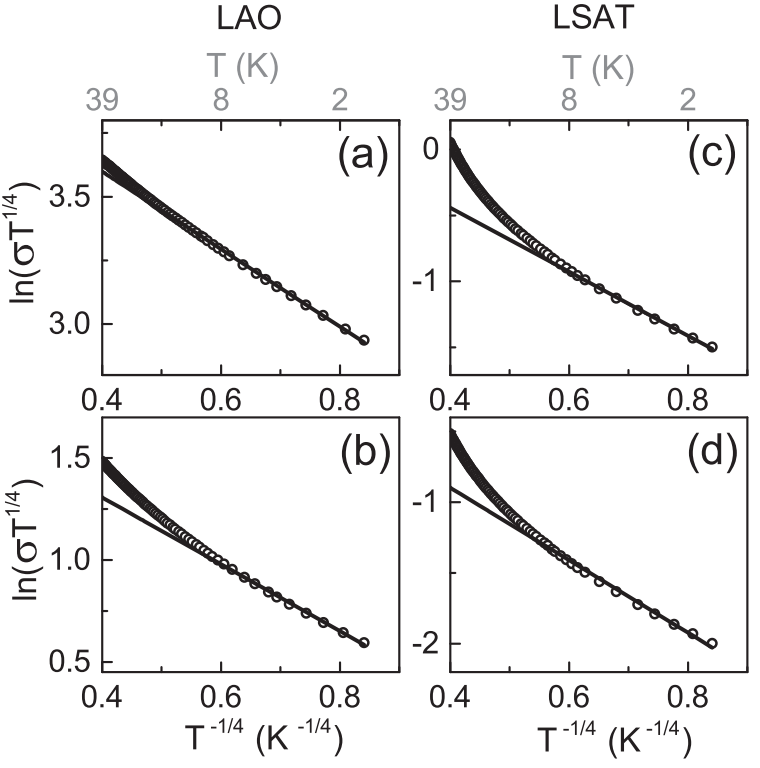

FIG. 4. Conductivity-temperature relationship $\ln \left(\sigma T^{1 / 4}\right)$ vs $T^{-1 / 4}$ in the NNO films on (a),(b) LAO and (c),(d) LSAT. Film thicknesses are (a),(c) $20 \mathrm{~nm}$ and (b),(d) $100 \mathrm{~nm}$. Straight lines show the fits to the Mott variable-range hopping model.

and 3(b)]. Similarly, the temperature $T_{\mathrm{MI}}$ is low, $T_{\mathrm{MI}} \approx$ 100-150 K, in the NNO/LSAT films [Figs. 3(c) and 3(d)]. However, inspection of the derivative $d \rho / d T$ (dashed curves in Fig. 3) reveals high-temperature metal-like behavior, which terminates at approximately $200 \mathrm{~K}$ in all films. For $T>200$ $\mathrm{K}$, a weak nearly linear decrease of the derivative with temperature agrees well with the previously reported resistivity behavior [expression (1)] in epitaxial NNO films [22]:

$$
\frac{1}{\rho(T)}=\frac{1}{\left(\rho_{0}+A T^{2}\right)}+\frac{1}{\rho_{\mathrm{SAT}}} .
$$

Here, $\rho_{\text {SAT }}$ is the saturation resistivity and the term $\left(\rho_{0}+\right.$ $A T^{2}$ ) describes a Landau Fermi liquid.

The metal behavior is evidenced only at temperatures above $\sim 200 \mathrm{~K}$, while a clear decrease of $d \rho / d T$ on cooling suggests a transition region around and below $200 \mathrm{~K}$. Concurrently, a low-temperature insulating phase is indicated by hopping conduction at temperatures $T \leqslant 15-20 \mathrm{~K}$. Assuming a Mott variable-range hopping [23,24], the conductivity $\sigma$ scales with temperature as (2)

$$
\sigma \propto T^{-1 / 4} \exp \left\{-\left(\frac{T_{0}}{T}\right)^{1 / 4}\right\}
$$

Good linear fits $\left[\ln \left(\sigma T^{1 / 4}\right) \propto T^{-1 / 4}\right]$ are obtained in all films at $T \leqslant 15-20 \mathrm{~K}$ (Fig. 4). The characteristic temperatures $T_{0}$ are extracted from the linear fits and equal approximately $T_{0} \approx 6-7 \mathrm{~K}$ and $T_{0} \approx 35-40 \mathrm{~K}$ in the films on LAO and LSAT, correspondingly. We note that changing the preexponent $T^{-1 / 4}$ in (2) to 1 or $T^{-1 / 2}$ leads to unphysically low or unreasonably high $T_{0}$ temperatures, respectively. 
The insulator phase with hopping conductivity is identified at temperatures significantly lower than $200 \mathrm{~K}$, above which the metal phase exists. These electrical observations indicate a possible coexistence of phases at intermediate temperatures. Recently, separate temperature-dependent regions of metal and insulator phases were detected by photoelectron emission microscopy in thin films of NNO [25]. Therefore, the conductivity of the NNO films can be considered that of a metal-insulator composite in a broad temperature range. Hence, inflection points in the temperature dependence of resistivity (Fig. 3) may rather correspond to a certain ratio of phase volumes than to an abrupt phase transition.

Here, we focus on the low-temperature insulating phase with hopping conduction. The related localized states, which are responsible for hopping, also lead to a strong magnetoresistance, as shown next.

\section{Magnetoresistance}

The measurements of conductivity $\sigma_{H}$ under an applied magnetic field at different fixed temperatures and during heating/cooling runs (field heating/field cooling) revealed a significant increase of conductivity compared to that without a magnetic field, $\sigma$. In more common terms of resistivity, a negative magnetoresistive effect is observed at low temperatures in all studied films. The magnetoresistance ratio (MR) $\Delta \rho / \rho=\left(\rho_{H}-\rho\right) / \rho$ (here, resistivity $\rho_{H}$ and $\rho$ are determined at a certain temperature with and without the magnetic field, respectively) is negative and up to several percent at $T<20 \mathrm{~K}$ (Fig. 5). The MR magnitude increases with the magnetic field and upon cooling. Additionally, the MR ratio varies with film thickness and the direction of the magnetic field. Also, MR is larger in the films on LSAT, which indicates a dependence on epitaxial strain.

The negative magnetoresistance (or $\sigma_{H}>\sigma$ ) of an insulator can be explained by Zeeman splitting of the localized states $[26,27]$. Considering that the localized states determine hopping conduction, the conductivity without a field is approximated by (3):

$$
\sigma=A \exp \left\{-\left(\frac{T_{0}}{T}\right)^{1 / n}\right\} .
$$

For each state, the magnetic field can produce spindependent Zeeman shifts $\Delta E_{Z}= \pm 1 / 2 g \mu_{\mathrm{B}} H$, where $g$ and $\mu_{\mathrm{B}}$ are the effective Lande factor and Bohr magneton, correspondingly. By neglecting interactions (between spins and/or charges) and tensor descriptions, a simplified model [26] suggests the transformation of expression (3) into (4) under applied field $H$ :

$$
\sigma_{H}=\frac{A}{2} \exp \left\{-\left(\frac{T_{+}}{T}\right)^{1 / n}\right\}+\frac{A}{2} \exp \left\{-\left(\frac{T_{-}}{T}\right)^{1 / n}\right\} .
$$

The new characteristic temperatures $T_{+}$and $T_{-}$are related to the temperature $T_{0}$ through the Fermi energy $E_{\mathrm{F}}$ and Zeeman shifts,

$$
T_{ \pm}=T_{0}\left(E_{\mathrm{F}} \pm \Delta E_{\mathrm{Z}}\right)
$$
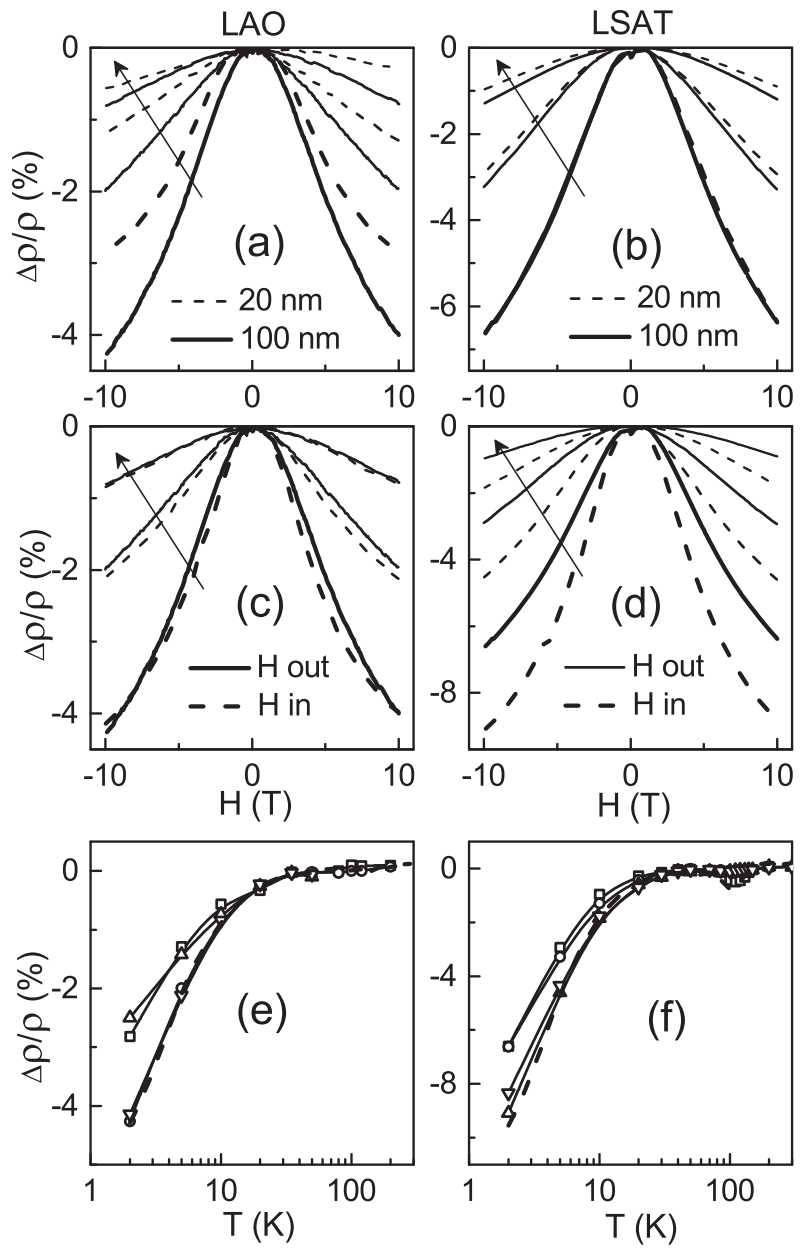

FIG. 5. The magnetoresistance ratio $\Delta \rho / \rho$ as a function of (a)-(d) magnetic field $H$ at different temperatures and as a function of (e),(f) temperature (note logarithmic $T$ scale) at $H=10 \mathrm{~T}$ in the NNO films on (a),(c),(e) LAO and (b),(d),(f) LSAT. Arrows show the direction of the temperature increase $T=2,5$, and $10 \mathrm{~K}$ in (a)-(d). Symbols and dashed curves in (e) and (f) correspond to the measurements at fixed temperatures and during field cooling, respectively.

For weak field-induced changes of the Fermi energy compared to $\Delta E_{Z}$, the relationship $\left(\sigma_{H}>\sigma\right)$ (or negative MR) directly follows from (4) and (5). Assuming $g=2$ and $n=$ 4 (Mott hopping), an approximate expression (6) can be obtained for $\sigma_{H}(H)$ under fields smaller than a critical field $H_{\mathrm{c}}(7)[26]$ :

$$
\begin{gathered}
\sigma_{H} \cong \sigma\left[1+\frac{9 \mu_{\mathrm{B}}^{2}}{32\left(E_{\mathrm{C}}-E_{\mathrm{F}}\right)^{2}} H^{2}\left(\frac{T_{0}}{T}\right)^{1 / 2}\right], \\
H_{\mathrm{c}}=\frac{4 \sqrt{2}}{3} \frac{\left(E_{\mathrm{C}}-E_{\mathrm{F}}\right)}{\mu_{\mathrm{B}}}\left(\frac{T}{T_{0}}\right)^{1 / 4} .
\end{gathered}
$$

The term $\left(E_{\mathrm{C}}-E_{\mathrm{F}}\right)$ describes the position of the Fermi level in relation to the conduction edge $E_{\mathrm{C}}$ in the band diagram in the case of $n$-type conductivity. For $p$-type conductivity, it corresponds to $\left(E_{\mathrm{F}}-E_{\mathrm{V}}\right)$. 

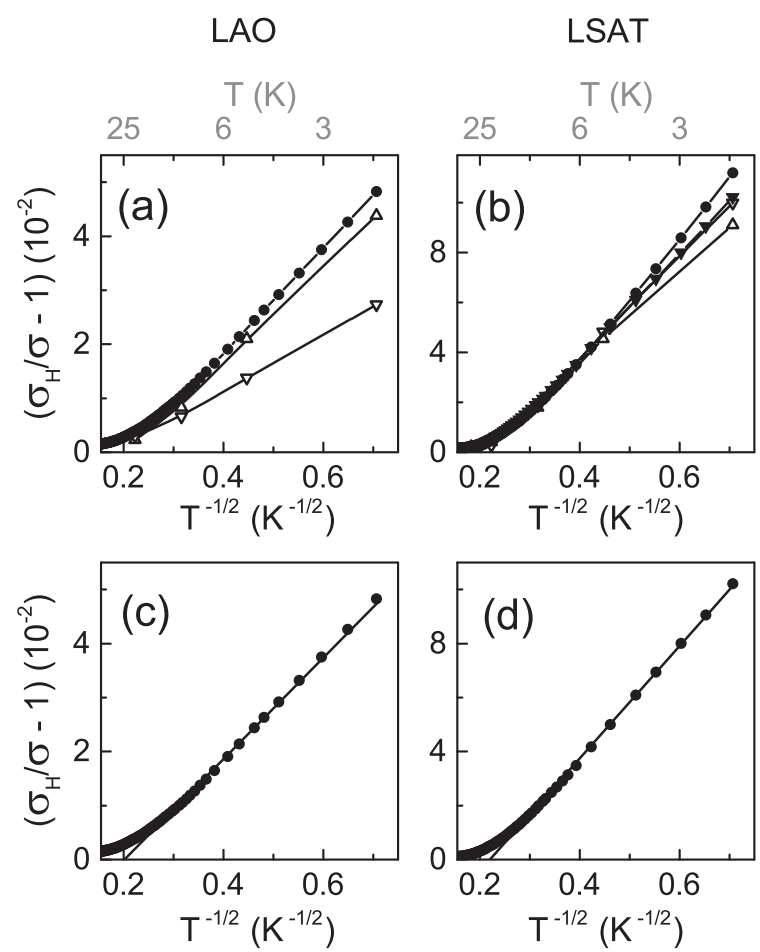

FIG. 6. Ratio $\left(\sigma_{H} / \sigma-1\right)$ as a function of $T^{-1 / 2}$ determined in the (20-100)-nm-thick films on (a),(c) LAO and (b),(d) LSAT at $H=10 \mathrm{~T}$, different fixed temperatures (open symbols), and in field cooling (solid symbols). Straight lines in (c) and (d) show fits.

For convenience of data analysis, we use the conductivity ratio $\left(\sigma_{H} / \sigma-1\right)$ :

$$
\begin{aligned}
\frac{\sigma_{H}}{\sigma}-1 & \cong A_{H} T^{-1 / 2}, \\
\frac{\sigma_{H}}{\sigma}-1 & \cong B_{\mathrm{T}} H^{2}, \\
H_{\mathrm{c}} & =C T^{1 / 4} .
\end{aligned}
$$

Thus, the conductivity ratio should be proportional to $T^{-1 / 2}$ for a fixed $H$ and proportional to $H^{2}$ for a fixed $T$ and fields $H<H_{\mathrm{c}}$, where the critical field $H_{\mathrm{c}}$ increases with $T^{1 / 4}$. The coefficients $A_{H}, B_{\mathrm{T}}$, and $C$ can be easily written using (6)-(10). To verify Zeeman splitting of the localized states as responsible for magnetoconduction, we investigate the relationships (8)-(10).

First, the experimentally determined ratio $\left(\sigma_{H} / \sigma-1\right)$ is plotted as a function of $T^{-1 / 2}$ in Fig. 6. Good linear fits are obtained for $T \leqslant 20 \mathrm{~K}$, validating expression (8). The slopes of $\left[\left(\sigma_{H} / \sigma-1\right) \propto T^{-1 / 2}\right]$ vary insignificantly depending on the measurement regime, while the effects of film thickness [Fig. 6(a)] and field direction [Fig. 6(b)] are seen.

Next, the field dependence of the ratio $\left(\sigma_{H} / \sigma-1\right)$ is analyzed [Figs. 7(a) and 7(b)]. The curves are of a complex nonparabolic shape, which is better revealed by the derivative $d\left(\left(\sigma_{H} / \sigma-1\right) / d H\right.$ [Figs. 7(c) and 7(d)]. The derivative is obtained numerically and using data smoothing by local regression methods. The model relationship (9) is proven by the linear behavior of the derivative $\left[d\left(\sigma_{H} / \sigma-1\right) / d H \propto H\right]$, which is clearly seen at fields $\left[-H_{\mathrm{c}-}<0<H_{\mathrm{c}+}\right]$. The critical fields are estimated as those at which the derivative exhibits
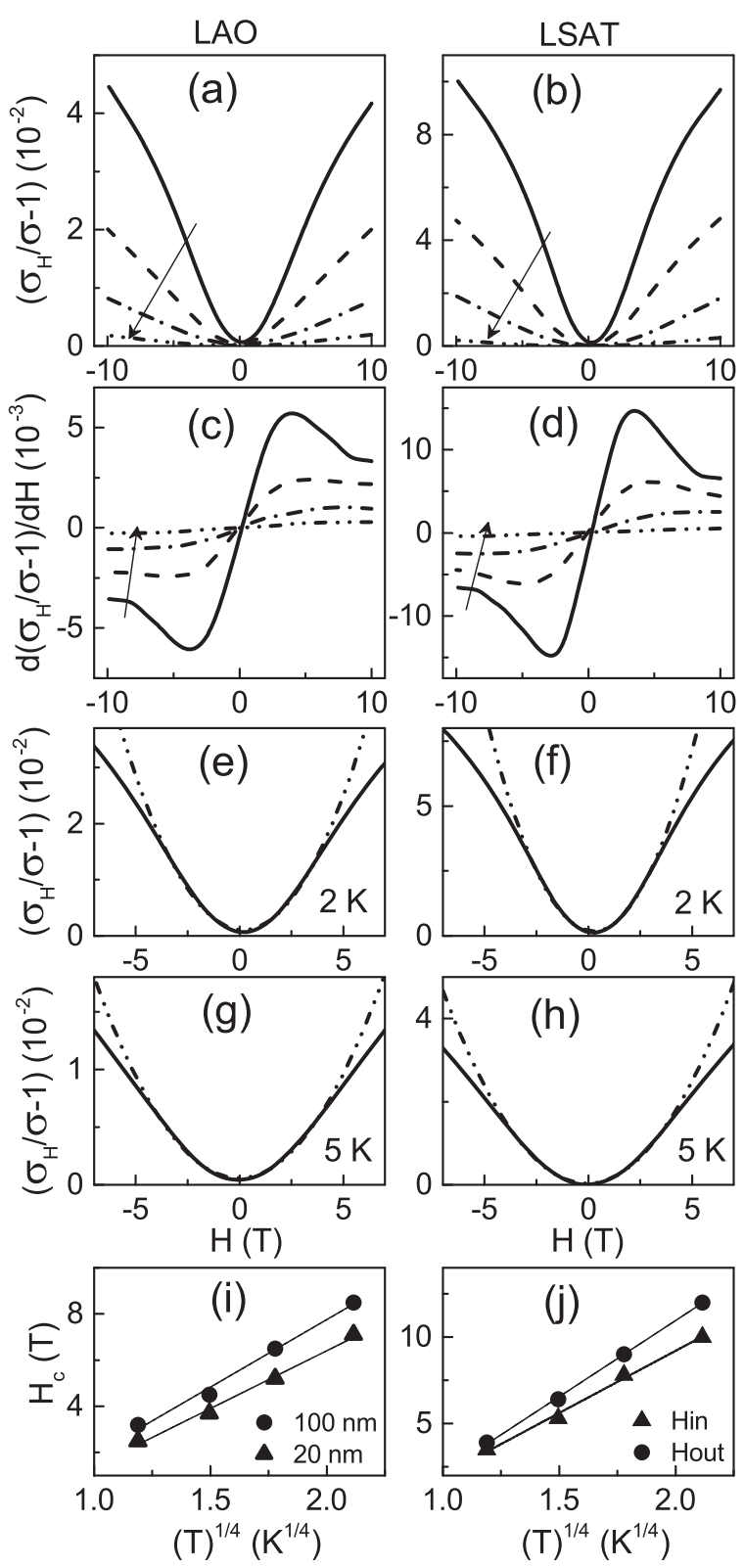

FIG. 7. (a),(b),(e)-(h) Ratio $\left(\sigma_{H} / \sigma-1\right)$ and (c),(d) its derivative as a function of field $H$ at different temperatures $T=2,5,10$, and 20 $\mathrm{K}$ in the 100-nm-thick films on (a),(c),(e),(g) LAO and (b),(d),(f),(h) LSAT. Arrows show the direction of the temperature increase in (a)(d). Dashed curves show parabolic fits in (e)-(h). (i),(j) The critical field $H_{\mathrm{c}}$ as a function of temperature in the films on (i) LAO and (j) LSAT.

anomalies [Figs. 7(c) and 7(d)]. The average critical field $H_{\mathrm{c}}=0.5\left(H_{\mathrm{c}-}+H_{c+}\right)$ increases with temperature [Figs. 7(i) and 7(j)], corroborating the model relationship (10). Good parabolic fits $\left[\left(\sigma_{H} / \sigma-1\right) \propto H^{2}\right]$ at fields $\left[-H_{\mathrm{c}-}<0<H_{\mathrm{c}+}\right]$ agree with the relationship (9) [Figs. 7(e)-7(h)].

\section{Localized states}

The results in Fig. 6 and Fig. 7 imply that the magneticfield-induced splitting of the localized states is responsible for the conductivity increase in the NNO films. In addition 

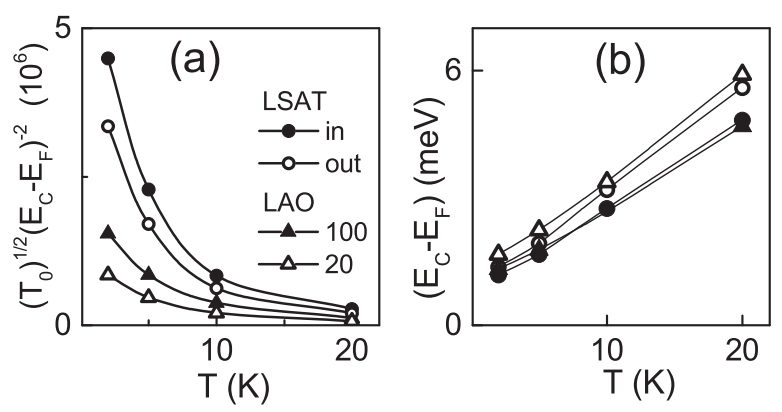

FIG. 8. Factor $\left(T_{0}\right)^{1 / 2}\left(E_{\mathrm{C}}-E_{\mathrm{F}}\right)^{-2}$ and width $\left(E_{\mathrm{C}}-E_{\mathrm{F}}\right)$ as a function of temperature in the NNO films.

to the temperature and field dependencies, the measured effect depends on film thickness (on LAO), field direction (on LSAT), and epitaxial strain [Figs. 5(a)-5(d)]. As seen from expression (6), the effect strength is determined by a filmspecific factor $\left[\left(T_{0}\right)^{1 / 2} \times\left(E_{\mathrm{C}}-E_{\mathrm{F}}\right)^{-2}\right]$. The temperature $T_{0}$, the energy width $\left(E_{\mathrm{C}}-E_{\mathrm{F}}\right)$, and the effect factor $\left[\left(T_{0}\right)^{1 / 2} \times\right.$ $\left.\left(E_{\mathrm{C}}-E_{\mathrm{F}}\right)^{-2}\right]$ were found from the fits to expressions (2) and (9) (Figs. 4, 6, and 7). The factor $\left[\left(T_{0}\right)^{1 / 2} \times\left(E_{\mathrm{C}}-E_{\mathrm{F}}\right)^{-2}\right]$ formally explains the observed variations in magnetoconductivity [Fig. 8(a)]. However, for the temperature $T_{0}$ of $6-40 \mathrm{~K}$ (or $0.5-3.5 \mathrm{meV}$ ) and the energy width $\left(E_{\mathrm{C}}-E_{\mathrm{F}}\right)>1 \mathrm{meV}$ [Fig. 8(b)], it is difficult to ascribe a dominating role to either $T_{0}$ or $\left(E_{\mathrm{C}}-E_{\mathrm{F}}\right)$ in the effect factor.

Remarkably, the width $\left(E_{\mathrm{C}}-E_{\mathrm{F}}\right)$ is found to increase with temperature in all films [Fig. 8(b)]. We note that inaccuracy in $T_{0}$ does not affect the revealed behavior. As mentioned above, the width $\left(E_{\mathrm{C}}-E_{\mathrm{F}}\right)$ describes the position of the Fermi level in relation to the conduction edge $E_{\mathrm{C}}$ in the band diagram. Because of $p$-type conduction in the epitaxial NNO films [16], the conduction edge corresponds to the topmost filled valence band (VB) and the Fermi level is located higher in energy, below the bottom of the conduction band (CB) [see schematics in Fig. 9(a)]. The detected temperature evolution of $\left(E_{\mathrm{C}}-E_{\mathrm{F}}\right)$ means that the Fermi level shifts upwards on heating. This observation implies that the density of localized states can increase on heating.

We suggest that the presence of localized states may be an intrinsic property of the insulating phase of NNO. As mentioned above, the insulating phase arises from the bond disproportionation and is characterized by the rock-salt order of the distinct small $\mathrm{Ni}_{\mathrm{S}} \mathrm{O}_{6}$ and large $\mathrm{Ni}_{\mathrm{L}} \mathrm{O}_{6}$ octahedra. At

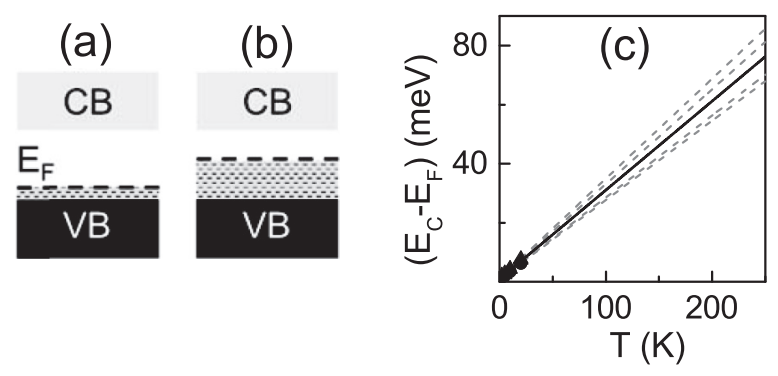

FIG. 9. (a),(b) Schematics of band diagrams with increasing temperature [from (a) to (b)]. (c) Extrapolated width $\left(E_{\mathrm{C}}-E_{\mathrm{F}}\right)$ as a function of temperature. The thick line shows average behavior. temperatures higher than $T=0$, the rock-salt $\mathrm{Ni}_{\mathrm{S}}: \mathrm{Ni}_{\mathrm{L}}$ order can be locally broken by thermally induced fluctuations of the $\mathrm{Ni}-\mathrm{O}$ lengths. The thermally stimulated disorder can produce localized states, whose density should grow with temperature as disorder increases. The behavior in Fig. 8(b) corroborates this scenario.

The thermally induced local disorder not only explains the localized states but also suggests a gradual evolution towards the metal phase on heating. A rough linear extrapolation of data in Fig. 8(b) shows that the Fermi level may shift by several tens of millielectronvolts with increasing temperature to $150-200 \mathrm{~K}$ [Fig. 9(b)]. Considering a gap $\Delta E_{\mathrm{g}}$ of $30-100 \mathrm{meV}$ in the insulating phase of the NNO films [28,29], the Fermi level may reach the conduction band at the temperatures when the macroscopic metal-range conduction becomes observable on heating (Fig. 3). This behavior of the Fermi level with the temperature increase is consistent with the optically detected filling of the gap [30] and mimics a gradual continuous closure of the gap.

The suggested thermally induced disorder can also lead to the nucleation and growth of conducting (metal) inclusions upon heating. As a result, the insulating and metal phases coexist in the broad temperature region around the macroscopic phase transition (Fig. 3). Interestingly, the order-disorder coexistence connotes NNO as a kind of "electronic relaxor" by its resemblance with perovskite relaxor ferroelectrics. Finally, we note that we found similar magnetoresistance behavior in the insulating phase of epitaxial (10-100 nm) films of $\mathrm{Sm}_{0.5} \mathrm{Nd}_{0.5} \mathrm{NiO}_{3}$ and $\mathrm{SmNiO}_{3}$. Although additional studies are desirable to clarify its origin unambiguously, we believe that local disorder plays an important role therein.

\section{CONCLUSIONS}

Cube-on-cube-type epitaxial perovskite NNO films of 20-100 nm in thickness are grown on (001) LAO and (001)LSAT substrates. The films exhibit hopping conductivity and large negative magnetoresistance in the insulating phase at low temperatures. The presence and Zeeman splitting of localized states are shown to be responsible for the observed behavior. The density of the localized states is found to increase with temperature. Therefore, the origin of the localized states is ascribed to thermally induced fluctuations of the Ni-O bond lengths that break the rock-salt order in the Ni-O bond disproportionation. The thermally induced local disorder is also suggested to lead to the phase coexistence in the broad temperature range around the macroscopic phase transition.

\section{ACKNOWLEDGMENTS}

The work was supported in part by the European Structural and Investment Funds and Ministry of Education, Youth and Sports of the Czech Republic through Programme "Research, Development and Education" (Project No. SOLID21 CZ.02.1.01/0.0/0.0/16 019/0000760). Research facilities of the Center for Advanced Functional Materials (SAFMAT) and the Materials Growth and Measurement Laboratory (MGML) were employed in this work. 
[1] M. L. Medarde, Structural, magnetic and electronic properties of $R \mathrm{NiO}_{3}$ perovskites ( $R=$ rare earth), J. Phys.: Condens. Matter 9, 1679 (1997).

[2] S. Catalano, M. Gibert, J. Fowlie, J. Íñiguez, J.-M. Triscone, and J. Kreisel, Rare-earth nickelates $\mathrm{RNiO}_{3}$ : Thin films and heterostructures, Rep. Prog. Phys. 81, 046501 (2018).

[3] H. Park, A. J. Millis, and C. A. Marianetti, Site-Selective Mott Transition in Rare-Earth-Element Nickelates, Phys. Rev. Lett. 109, 156402 (2012).

[4] S. Johnston, A. Mukherjee, I. Elfimov, M. Berciu, and G. A. Sawatzky, Charge Disproportionation without Charge Transfer in the Rare-Earth-Element Nickelates as a Possible Mechanism for the Metal-Insulator Transition, Phys. Rev. Lett. 112, 106404 (2014).

[5] A. Subedi, O. E. Peil, and A. Georges, Low-energy description of the metal-insulator transition in the rare-earth nickelates, Phys. Rev. B 91, 075128 (2015).

[6] J. Varignon, M. N. Grisolia, J. Íñiguez, A. Barthélémy, and M. Bibes, Complete phase diagram of rare-earth nickelates from first-principles, npj Quantum Mater. 2, 21 (2017).

[7] A. S. Everhardt, S. Matzen, N. Domingo, G. Catalan, and B. Noheda, Ferroelectric domain structures in low-strain $\mathrm{BaTiO}_{3}$, Adv. Electron. Mater. 2, 1500214 (2016).

[8] A. R. Damodaran, E. Breckenfeld, Z. Chen, S. Lee, and L.W. Martin, Enhancement of ferroelectric curie temperature in $\mathrm{BaTiO}_{3}$ films via strain-induced defect dipole alignment, Adv. Mater. 26, 6341 (2014).

[9] J. L. Garcia-Munoz, J. Rodriguez-Carvajal, P. Lacorre, and J. B. Torrance, Neutron-diffraction study of $\mathrm{RNiO}_{3} \quad(R=$ $\mathrm{La}, \mathrm{Pr}, \mathrm{Nd}, \mathrm{Sm}$ ): Electronically induced structural changes across the metal-insulator transition, Phys. Rev. B 46, 4414 (1992).

[10] A. Vailionis, H. Boschker, W. Siemons, E. P. Houwman, D. H. A. Blank, G. Rijnders, and G. Koster, Misfit strain accommodation in epitaxial $A B \mathrm{O}_{3}$ perovskites: Lattice rotations and lattice modulations, Phys. Rev. B 83, 064101 (2011).

[11] I. C. Tung, P. V. Balachandran, J. Liu, B. A. Gray, E. A. Karapetrova, J. H. Lee, J. Chakhalian, M. J. Bedzyk, J. M. Rondinelli, and J. W. Freeland, Connecting bulk symmetry and orbital polarization in strained $R \mathrm{NiO}_{3}$ ultrathin films, Phys. Rev. B 88, 205112 (2013).

[12] A. K. Choquette, C. R. Smith, R. J. Sichel-Tissot, E. J. Moon, M. D. Scafetta, E. Di Gennaro, F. Miletto Granozio, E. Karapetrova, and S. J. May, Octahedral rotation patterns in strained $\mathrm{EuFeO}_{3}$ and other Pbnm perovskite films: Implications for hybrid improper ferroelectricity, Phys. Rev. B 94, 024105 (2016).

[13] D. Kan, Y. Wakabayashi, H. Tajiri, and Y. Shimakawa, Interfacially engineered oxygen octahedral rotations and their impact on strain relief in coherently grown $\mathrm{SrRuO}_{3}$ films, Phys. Rev. B 94, 024112 (2016).

[14] J. Fowlie, M. Gibert, G. Tieri, A. Gloter, J. Íñiguez, A. Filippetti, S. Catalano, S. Gariglio, A. Schober, M. Guennou, J. Kreisel, O. Stéphan, and J.-M. Triscone, Conductivity and local structure of $\mathrm{LaNiO}_{3}$ thin films, Adv. Mater. 29, 1605197 (2017).
[15] G. Catalan, R. M. Bowman, and J. M. Gregg, Transport properties of $\mathrm{NdNiO}_{3}$ thin films made by pulsed-laser deposition, J. Appl. Phys. 87, 606 (2000).

[16] R. Scherwitz, P. Zubko, I. G. Lezama, S. Ono, A. F. Morpurgo, G. Catalan, and J.-M. Triscone, Electric Field Control of the Metal-Insulator Transition in Ultrathin $\mathrm{NdNiO}_{3}$ Films, Adv. Mater. 22, 5517 (2010).

[17] J. Hwang, J. Y. Zhang, J. Son, and S. Stemmer, Nanoscale quantification of octahedral tilts in perovskite films, Appl. Phys. Lett. 100, 191909 (2012).

[18] Y. Kumar, R. J. Choudhary, S. K. Sharma, M. Knobel, and R. Kumar, Strain dependent stabilization of metallic paramagnetic state in epitaxial $\mathrm{NdNiO}_{3}$ thin films, Appl. Phys. Lett. 101, 132101 (2012).

[19] P.-H. Xiang, N. Zhong, C.-G. Duan, X. D. Tang, Z. G. Hu, P. X. Yang, Z. Q. Zhu, and J. H. Chu, Strain controlled metalinsulator transition in epitaxial $\mathrm{NdNiO}_{3}$ thin films, J. Appl. Phys. 114, 243713 (2013).

[20] L. Wang, S. Ju, L. You, Y. Qi, Y. Guo, P. Ren, Y. Zhou, and J. Wang, Competition between strain and dimensionality effects on the electronic phase transitions in $\mathrm{NdNiO}_{3}$ films, Sci. Rep. 5, 18707 (2015).

[21] J. Y. Zhang, H. Kim, E. Mikheev, A. J. Hauser, and S. Stemmer, Key role of lattice symmetry in the metal-insulator transition of $\mathrm{NdNiO}_{3}$ films, Sci. Rep. 6, 23652 (2016).

[22] E. Mikheev, A. J. Hauser, B. Himmetoglu, N. E. Moreno, A. Janotti, C. G. Van de Walle, and S. Stemmer, Tuning bad metal and non-Fermi liquid behavior in a Mott material: Rare-earth nickelate thin films, Sci. Adv. 1, e1500797 (2015).

[23] N. Tsuda, K. Nasu, A. Fujimori, and K. Siratori, Electronic Conduction in Oxides (Springer, Berlin, 2000).

[24] N. F. Mott and E. A. Davis, Electronic Processes in NonCrystalline Materials (Oxford University Press, Oxford, 2012).

[25] G. Mattoni, P. Zubko, F. Maccherozzi, A. J. H. van der Torren, D. B. Boltje, M. Hadjimichael, N. Manca, S. Catalano, M. Gibert, Y. Liu, J. Aarts, J.-M. Triscone, S. S. Dhesi, and A. D. Caviglia, Striped nanoscale phase separation at the metalinsulator transition of heteroepitaxial nickelates, Nat. Commun. 7, 13141 (2016)

[26] H. Fukuyama and K. Yoshida, Negative Magnetoresistance in the Anderson Localized States, J. Phys. Soc. Jpn. 46, 102 (1979).

[27] K. Ramadoss, N. Mandal, X. Dai, Z. Wan, Y. Zhou, L. Rokhinson, Y. P. Chen, J. Hu, and S. Ramanathan, Sign reversal of magnetoresistance in a perovskite nickelate by electron doping, Phys. Rev. B 94, 235124 (2016).

[28] M. K. Stewart, D. Brownstead, J. Liu, M. Kareev, J. Chakhalian, and D. N. Basov, Heterostructuring and strain effects on the infrared optical properties of nickelates, Phys. Rev. B 86, 205102 (2012).

[29] S. J. Allen, A. J. Hauser, E. Mikheev, J. Y. Zhang, N. E. Moreno, J. Son, D. G. Ouellette, J. Kally, A. Kozhanov, L. Balents, and S. Stemmer, Gaps and pseudogaps in perovskite rare earth nickelates, APL Mater. 3, 062503 (2015).

[30] M. K. Stewart, J. Liu, M. Kareev, J. Chakhalian, and D. N. Basov, Mott Physics near the Insulator-To-Metal Transition in $\mathrm{NdNiO}_{3}$, Phys. Rev. Lett. 107, 176401 (2011). 